\title{
OBSERVATION OF TWO-ELECTRON-ONE-PHOTON TRANSITIONS IN SILICON *
}

\author{
B. KNAF, G. PRESSER and J. STÄHLER \\ Institut für Physik, Universität Dortmund, D-4600 Dortmund 50, Germany \\ and \\ B. FRICKE \\ Gesamthochschule Kassel, D-3500 Kassel, Germany
}

Received 15 October 1976

\begin{abstract}
We report on the observation of $\mathrm{K} \alpha \alpha \mathrm{X}$-rays of $\mathrm{Si}$, produced in collisions of $15-28 \mathrm{MeV}$ Si projectiles with va-rious target atoms in the range $Z=6$ to 29 . Energy shifts of $X$-rays were measured and are compared with theoretical predictions. Cross section ratios for emission of $\mathrm{K} \alpha \alpha$ and $\mathrm{K} \alpha$ radiation are given.
\end{abstract}

Since the first experimental identification of cooperative two-electron transitions in the $\mathrm{K}$ shell of doubly ionized atoms leading to the emission of a single photon, published recently in a paper ty Wölfli et al. [1], increasing interest has been devoted to the study of these phenomena. Further experimental work [2-5] has confirmed the existence of these transitions in several atoms. Measured K $\alpha \alpha \mathrm{X}$-ray energies are found to be in rather good agreement with theoretical calculations [6--9], whereas relative transition rates are still subject of some theoretical controversy [9-11]. A convenient method for study of two-electron-one-photon transitions is the observation of $\mathrm{X}$ rays induced in collisions between heavy ions and atoms because of the relatively large probability for the production of two $\mathrm{K}$ shell vacancies in such reactions [12]. We have investigated two-electron-onephoton transitions into $\mathrm{K}$ shell of silicon, measuring energies of $\mathrm{K} \alpha$ and $\mathrm{K} \alpha \alpha \mathrm{X}$-rays and production cross sections for various target atomic numbers and several incident silicon projectile energies and present comparison with calculated X-ray energies.

Experiments were performed with the ${ }^{28} \mathrm{Si}$ beam from the $4 \mathrm{MV}$ Dynamitron-Tandem at Bochum. Thin targets (effective thickness of $20-60 \mu \mathrm{g} / \mathrm{cm}^{2}$ ) either self-supporting foils $(\mathrm{C}, \mathrm{Al})$ or evaporated onto $20 \mu \mathrm{g} / \mathrm{cm}^{2}$ carbon backings ( $\mathrm{Si}, \mathrm{KBr}, \mathrm{Ti}, \mathrm{Fe}, \mathrm{Cu}$ ) were bombarded with $15-28 \mathrm{MeV}^{28} \mathrm{Si}$ ions. X-rays emitted

* Work partially supported by GSI, Darmstadt, Germany.

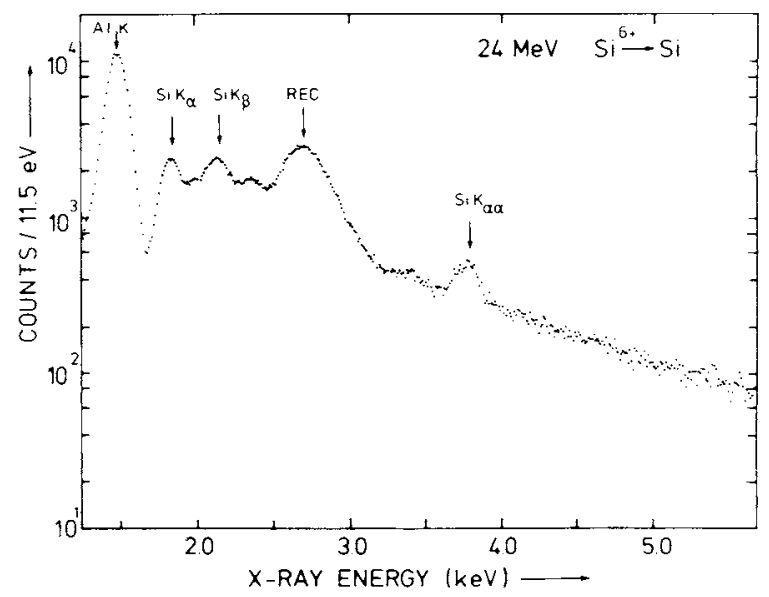

Fig. 1. Observed X-ray spectrum from $24 \mathrm{MeV} \mathrm{Si}^{6+}$ bombardment of a $15 \mu \mathrm{g} / \mathrm{cm}^{2}$ Si target. The spectrum is not corrected for absorption, which is mainly due to a $10 \mu \mathrm{m} \mathrm{Al}$ foil. Fuorescence excited absorber $\mathrm{X}$-rays, characteristic $\mathrm{K} \alpha$ and $\mathrm{K} \beta$ radiation of $\mathrm{Si}$ and $\mathrm{X}$-rays from radiative electron capture into projectile $\mathrm{K}$-shell are clearly visible. The single peak at 3.75 $\mathrm{keV}$ is attributed to the two-electron--one-photon transition into the Si $\mathrm{K}$-shell. It is imposed on an almost exponentially decreasing background of non-characteristic radiation.

from projectiles and target atoms were observed at $90^{\circ}$ with respect to the beam line through a $6 \mu \mathrm{m}$ Mylar window separating the vacuum chamber from a $3 \mathrm{~mm}$ $\times 10 \mathrm{~mm}^{2} \mathrm{Si}(\mathrm{Li})$ detector with an energy resolution of $158 \mathrm{eV}$ at $5.9 \mathrm{keV}$. For measurement of K $\alpha \alpha$ intensities, low energy X-rays (mainly Si K $\alpha$ ) were strongly 


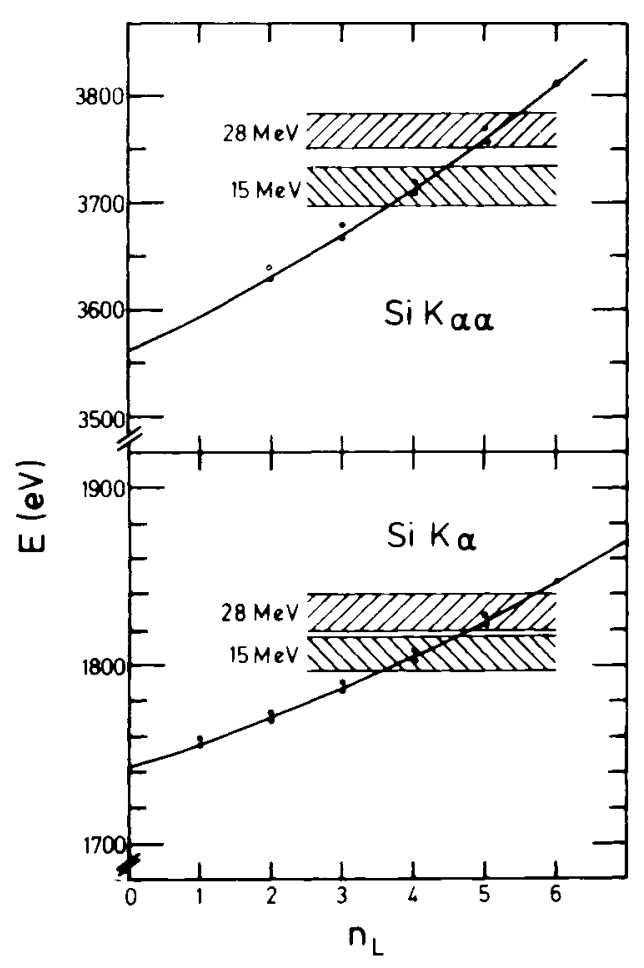

Fig. 2. Comparison of measured $K \alpha$ and $K \alpha \alpha$ energies of silicon with calculations using a Dirac-Fock-Slater program [14]. The shaded bands represent experimental X-ray energies for two projectile energies. Calculations were performed for different $\mathrm{L}$-shell defect configurations. Theoretical energies are plotted versus $n_{\mathrm{L}}$, the number of $\mathrm{L}$ shell vacancies accompanying the $\mathrm{K}$-shell vacancies and are connected by solid lines. Different points at the same $n_{L}$ represent different combinations of subshell holes. Additional $M$-shell vacancies change the calculated $\mathrm{X}$-ray energies by less than the experimental uncertainties.

suppressed by absorber foils to avoid pile-up effect in the vicinity of the $K \alpha \alpha$.line. Projectile and target $K \alpha$ and $\mathrm{K} \beta \mathrm{X}$-ray cross sections were measured separately without absorber foils to reduce necessary correction factors in cross section evaluation. Normalization to incoming particle flux and target thickness was achieved with Rutherford scattered projectiles observed at $30^{\circ}$ scattering angle by a surface barrier detector. In fig. 1 is shown an uncorrected $\mathrm{X}$-ray spectrum from the symmetric collision system $\mathrm{Si}+\mathrm{Si}$ at $24 \mathrm{MeV}$ bombarding energy. The $\mathrm{Si} K \alpha$ and $K \beta$ peaks are well separated due to their large energy shifts of $80 \mathrm{eV}$ and $270 \mathrm{eV}$ respectively. Radiative electron capture [13] is producing the broad peak centred near $2.7 \mathrm{keV}$. The peak

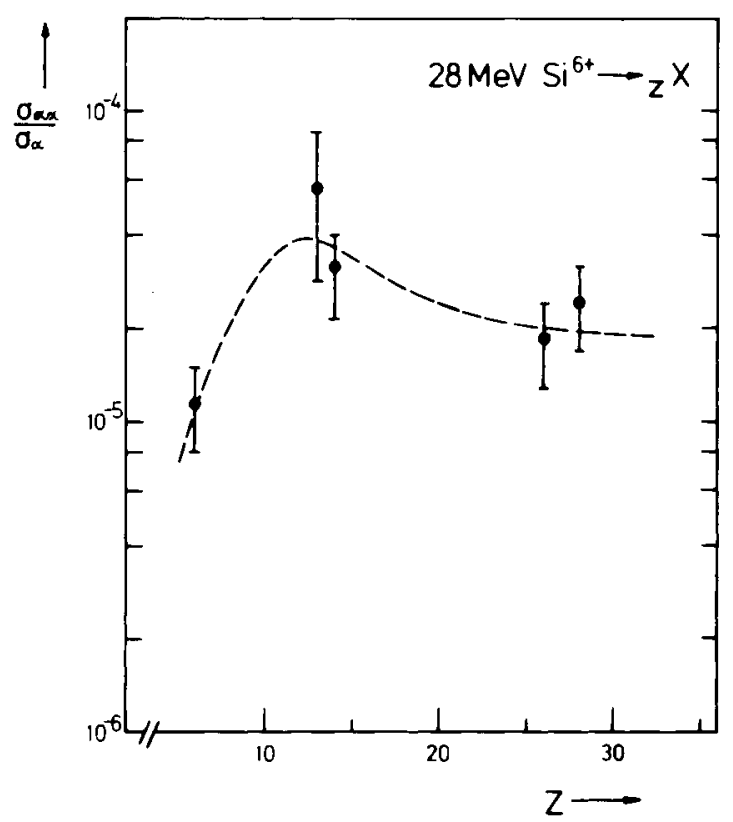

Fig. 3. Ratio of cross sections for production of Si K $\alpha \alpha$ and $\mathrm{K} \alpha \mathrm{X}$-rays as function of atomic number $Z$ of target atoms. The broken curve is arbitrarily drawn through data points to indicate the gross behaviour of the ratio with $Z$. Similar dependence is found for the other projectile energies.

at $3.75 \mathrm{keV}$ is ascribed to the two-electron-one-photon transition in Si atoms with two K-shell vacancies. Its identification is based on the following experimental observations: (i) the peak is observed in bombardment of all targets, except $\mathrm{KBr}$ and $\mathrm{Ti}$ where characteristic $\mathrm{X}$-rays of target atoms obscure its identification; (ii) pile-up effect could be excluded by measurements with different absorber thicknesses; (iii) the peak is clearly shifted from twice the measured $\mathrm{Si} \mathrm{K} \alpha$ energy by a positive amount of $103 \pm 10 \mathrm{eV}$; (iv) the Al target was subsequently checked for the presence of impurities by the method of proton induced $\mathrm{X}$-ray analysis giving an upper limit for the concentration of trace elements with characteristic X-rays in the interesting region of at least two orders of magntidue too low to explain the peak found in the measurements with $\mathrm{Si}$ projectiles. The experimental observations are in full agreement with the assumption that the peak is due to the two-electron-one-photon transition.

Energies of $K \alpha, K \beta$ and $K \alpha \alpha$ are influenced by vacancies in outer shells [5-9]. Calculations of these energies were performed with a Dirac-Fock-Slater [14] and a Dirac-Fock [15] program for various outer 
sheli vacancy configurations assuming the $K \alpha \alpha$ line as beir.g due to a $1 s^{-2} \rightarrow 2 s^{-1} 2 p^{-1}$ electric dipole transition $[2,7-9]$. The two different computation methods lead within less than $7 \mathrm{eV}$ to the same transition energies. In fig. 2 are shown the results for $E_{\alpha}$ and $E_{\alpha \alpha}$ from calculations as function of $n_{L}$, the number of additional $L$ shell vacancies accompanying the 1 s vacancies. The experimental values of $\mathrm{K} \alpha$ and $\mathrm{K} \alpha \alpha$ energies are in good agreement with $n_{\mathrm{L}}=5 \pm 1$ at 28 $\mathrm{MeV}$ and $n_{\mathrm{L}}=4 \pm 1$ at $15 \mathrm{MeV}$. The same $n_{\mathrm{L}}$ are also obtained from calculated and measured $\mathrm{K} \beta$ energy shifts. Furthermore the mean charge state of $\mathrm{Si}$ ions after penetration of a solid foil, $\vec{q}=10$ at $28 \mathrm{MeV}$ and $\bar{q}=$ 8.5 at $15 \mathrm{MeV}$ [16] is consistent with the obtained $n_{\mathrm{L}}$ if the Si M Shell is assumed to be almost completely stripped. The experimental K $\alpha \alpha$ energy increases by about $60 \mathrm{eV}$ with bombarding energy varying between 15 and $28 \mathrm{MeV}$, however, the difference $E_{\alpha \alpha}(\exp )-$ $2 E_{\alpha}(\exp )$ is found to be constant within the experimental uncertainty of $\pm 10 \mathrm{eV}$, reflecting the similar influence of outer shell vacancies on $E_{\alpha}$ and $E_{\alpha \alpha}[7]$. The width of the K $\alpha \alpha$ peak shows no additional broadening as was observed fro neighbouring $K \alpha$ and $K \beta$ radiation of $K$ and $T i$ induced by collisions with $\mathrm{Si}$ ions. The observed $\mathrm{K} \alpha \alpha$ width is completely due to detector energy resolution.

Because all measurements were performed with thin targets, cross sections could easily be deduced from $X$-ray yields, the known detector efficiency including absorption corrections and solid angles, and the yield of Rutherford-scattered projectiles. Fig. 3 shows cross section ratios for production of $\mathrm{K} \alpha \alpha$ and $\mathrm{K} \alpha \mathrm{X}$-rays plotted versus the atomic number $Z$ of the target. The ratio has its maximum of $4 \times 10^{-5}$ for symmetric collisions and decreases with increasing deviation from symmetry, a behaviour that is to be expected if vacancy production via molecular orbital mechanisms [17] is dominating. Similar observations were reported for other collision systems [3]. The cross section ratio for all investigated collision systems is found to be by a factor of 2 smaller at $15 \mathrm{MeV}$ bombarding energy, whereas the $\mathrm{Si} K \alpha \alpha$ cross section itself decreases by one order of magnitude from 28 to $15 \mathrm{MeV}$. Observation of $\mathrm{K} \alpha \alpha \mathrm{X}$-rays in experiments with thin targets offers an excellent method to investigate the mechanism of multiple vacancy creation in inner atomic shells. Further experiments to study in more detail projectile energy and target atomic number dependence of $\mathrm{K} \alpha \alpha$ cross sections are now in progress.

We would like to thank K. Brand and his coworkers for the skillful operation of the accelerator and Mrs. I. Kamphausen for the preparation of targets.

\section{References}

[1] W. Wölfli et al., Phys. Rev. Lett. 35 (1975) 656.

[2] Th.P. Hoogkaner et al., J. Phys. B9 (1976) L145.

[3] R. Schuch et al., Contributed paper 2nd Int. Conf. on Inner shell ionization phenom. (Freiburg 1976) p. 161.

[4] Ch. Stoller et al., Phys. Lett. 58A (1976) 18.

[5] A.R. Knudson et al., Phys. Rev. Lett. 37 (1976) 679.

[6] D.J. Nagel et al., Phys. Rev. Lett. 36 (1976) 164.

[7] J.P. Briand, Phys. Rev. Lett. 37 (1976) 59.

[8] W. Wölfli and H.D. Betz, Phys. Lett. 37 (1976) 61.

[9.] T. Aberg, K.A. Jamison and P. Richard, Phys. Rev. Lett. 37 (1976) 63.

[10] H.P. Kelly, Phys. Rev. Lett. 37 (1976) 386.

[11] H. Nussbauer, J. Phys. B9 (1976) 1757.

[12] P. Richard, W. Hodge and C.I. Moore, Phys. Rev. Lett. 29 (1972) 393.

[13] H.W. Schnopper et al., Phys. Rev. Lett. 29 (1972) 898.

[14] B. Fricke, W. Greiner and J.T. Waber, Theor. Chim. Acta 21 (1971) 235.

[15] J.P., Desclaux, Atomic Data Nucl. Data Tab. 12 (1973) 311.

[16] H.D. Betz, Rev. Mod. Phys. 44 (1972) 465.

[17] M. Barat and W. Lichten, Phys. Rev. A6 (1972) 211. 